\title{
REV UE
}

\section{INTERNATIONA LE}

DE LA

CROIX-ROUGE

ET

BULLETIN INTERNATIONAL

DES

SOCIETES DE LA CROIX-ROUGE

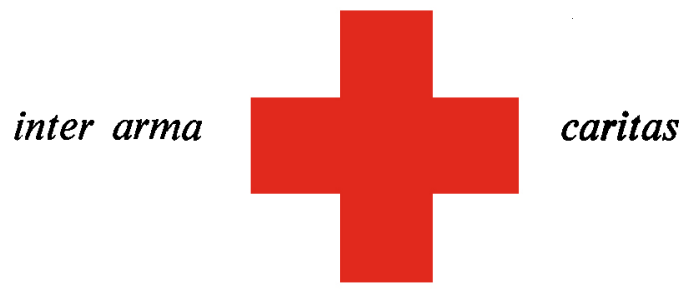




\title{
COMITÉ INTERNATIONAL DE LA CROIX-ROUGE
}

\author{
à Genève
}

MM. MAX HUBER, docteur en droit, ancien président de la Cour permanente de justice internationale, président d'honneur (1923) ${ }^{1}$

PAUL. RUEGGER, docteur en droit, ancien ministre de Suisse en Italie et en Grande-Bretagne, président (1948)

JACQUES CHENEVIERE, docteur ès lettres h. c. (1919)

M1le LUCIE ODIFR, ex-chef du Service des infirmières-visiteuses de la section genevoise de la Croix Rouge suisse (1930)

MM. CARL J. BURCKHARDT, docteur en philosophie, ancien ministre de Suisse en France (1933)

ÉDOUARD CHAPUISAT, docteur ès lettres h. c. (1938)

MARTIN BODMER, docteur en philosophie h. c. (1940)

ERNEST GLOOR, docteur en médecine, vice-président (1945)

RENÊ VAN BERCHEM, docteur en droit, banquier (1946)

LEOPOLD BOISSIER, docteur en droit, professeur à l'Université de Genève, ancien secrétaire général de l'Union interparjementaire, vice-présudent (1946)

PAUL CARRY, docteur en droit, professeur à l'Université de Genève (1946)

EIMOND GRASSET, docteur en médecine, professeur à l'Université de Genève, directeur de l'Institut d'hygiène (1946)

HENRI GUISAN, général, ancien commandant en chef de l'armée suisse (1948)

ALFREDO VANNOTTI, docteur en médecine, professeur à l'Université de Lausanne (1949)

RODOLFO OLGIATI, ancien directeur du Don suisse (1949)

Mile MARGUERITE VAN BERCHEM, ancien chef de service de l'Agence centrale des prisonniers de guerre (1951)

MM. FRÉDÉRIC SIORDET, avocat, conseiller du Comité international de la Croix-Rouge depuis 1943 (1951)

MARCEL JUNOD, docteur en médecine, délégué du Comité international de la Croix-Rouge de 193. à 1946 (1952)

ERNEST NOBS, ancien conseiller fédéral (1952)

GUILlAUME BORDIER, ingénieur dipl. E.P.F., M.B.A. Harvard, banquier (1955)

\section{Direction :}

MM. ROGER GALLOPIN, directeur exécutif

JEAN S. PICTET, directeur des Affaires générales

DAVID DE TRAZ, directeur exécutif-adjoint

ÉDOUARD DE BONDELI, sous-directeur

CLAUDE PILLOUD, sous-đirecteur des Affaires générales

${ }^{1}$ Les années indiquées dans les parenthèses désignent ies dates de nomination des membres du Comité international. 


\section{REVUE INTERNATIONALE \\ DE LA CROIX-ROUGE}

publiée, à la fin de chaque mois, par le Comité international de la Croix-Rouge

7, avenue de la Paix, Genève (Suisse) - Compte de chèques postaux I. 1767

Abonnement un an : Fr. 20.- ; le numéro Fr. 2.-

Rédaction : JEAN-G. LOSSIER

TRENTE-SEPTIĖME ANNEE - N. 436

AVRIL 1955

SOMMAIRE

\section{COMITÉ INTERNATIONAL DE LA CROIX-ROUGE}

Activités diverses: Le CICR à Costa-Rica (R.M.) . . . . . . 223

Nouvelles brèves. . . . . . . . . . . . . . . 227

Fonds de l'Impératrice Shôken (trente-quatrième distribution des revenus)................ . . . . 232

Fondation en faveur du CICR: Situation et compte de résultats au 31 décembre 1954 . . . . . . . . . . . . . . 235

Fonds Augusta et Fonds de la Médaille Florence Nightingale: situation au 31 décembre I954 . . . . . . . . . . . 237

Les hôtes du CICR . . . . . . . . . . . . . . 239

\section{CROIX-ROUGE INTERNATIONALE}

Réunion des trois présidents . . . . . . . . . . . . 240

Le soldat sanitaire n'est pas un combattant (J.P.S.) . . . . . . $24 \mathrm{I}$

Général-Médecin Jules Voncken : Le médecin, un combattant ? . . . 245

Deux précurseurs : Saladin et Açoka . . . . . . . . . . 250

\section{CHRONIQUE}

Diffusion des Conventions de Genève de 1949 (J. de Preux) . . .

\section{BIBLIOGRAPHIE}

Legal Aid in the United States, par Emery A. Brownell (H.C.)

\section{A TRAVERS LES REVUES}




\title{
BULLETIN INTERNATIONAL
}

\section{DES SOCIÉTES DE LA CROIX-ROUGE}

*

\section{SOMMAIRE}

\begin{abstract}
Pages
Allemagne (Republigue fédérale) . . . . . . . . . . . 275

Etats-Unis . . . . . . . . . . . . . . . . . . . . 276

ISRAËL . . . . . . . . . . . . . . . . . . . . . 278

Philippines . . . . . . . . . . . . . . . . . . 283

SuÈdE . . . . . . . . . . . . . . . . . . . . . 285
\end{abstract}

\section{SUPPLEMENTS DE LA REVUE}

EN LANGUE ANGLAISE

Ninth Distribution of the Revenue of the Augusta Fund (Circular No. 407). - Comments of a delegate of the International Committee of the Red Cross on his return from Indo-China. - Chronicle : The dissemination of the Geneva Conventions of 1949 ( $\mathrm{J}$. de Preux).

EN LANGUE ESPAGNOLE

Novena distribución de rentas del Fondo a Augusta " (Circular no 407). Al regresar de la Indochina. - Crónica : Difusión de los Convenios de Ginebra (J. de Preux).

\section{EN LANGUE ALLEMANDE}

Neunte Verteilung der Einkünfte aus dem Fonds Augusta (4\%7. Rundschreiben). - Bericht eines Delegierten des Internationalen Komitees nach seiner Rückkehr aus Indochina. - Chronik : Verbreitung der Genfer Abkommen von 1949 (J. de Preux). 


\section{Votre Banque...}

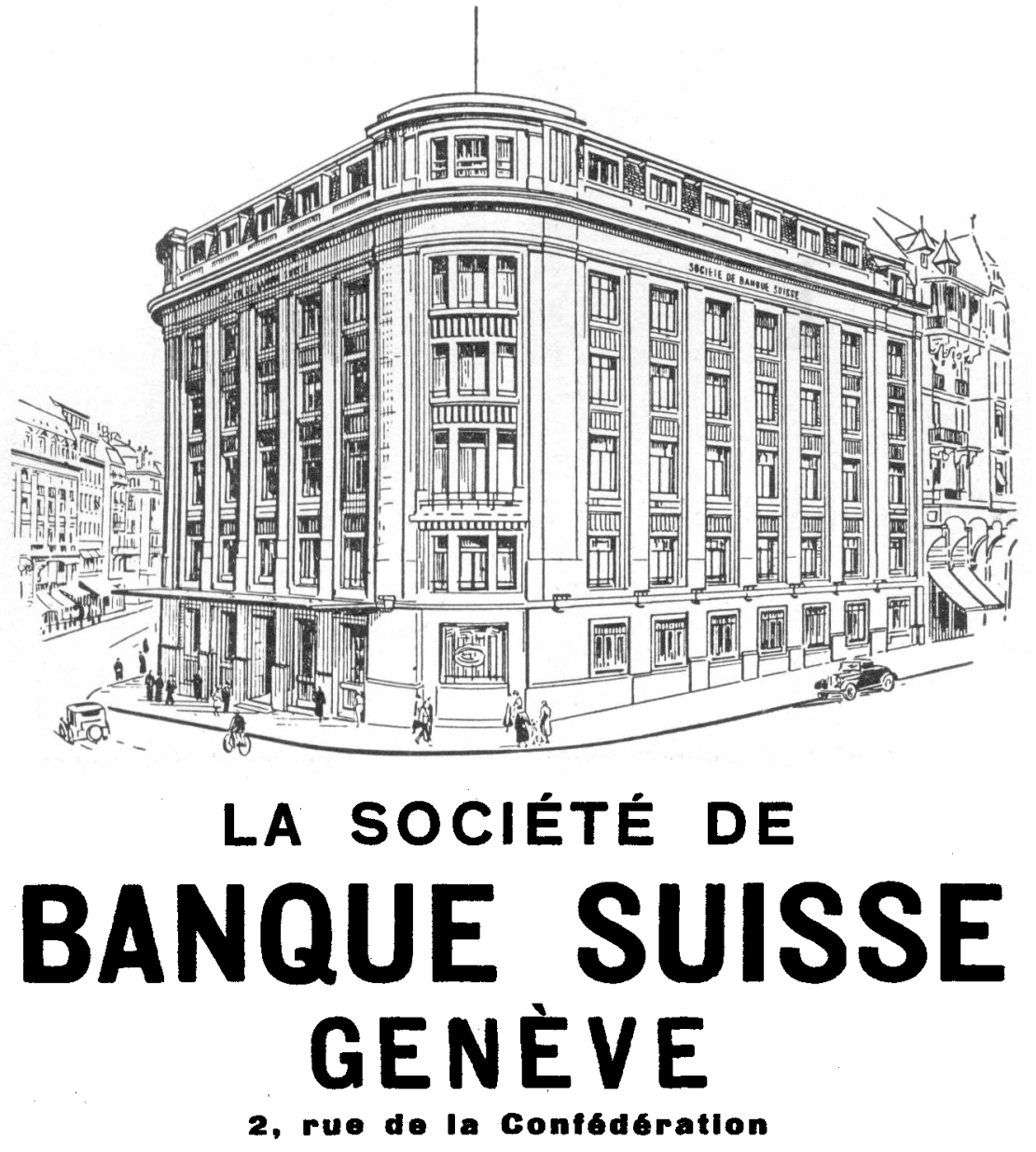

BALE ZURIOH ST-GALL LAUSANNE NEUCHATEL CHAUX-DE-FONDS SCHAFFHOUSE BIENNE LONDRES NEW-YOKR

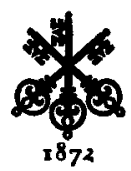

CAPITAL ET RESERVES FRS 35 MILLIONS 


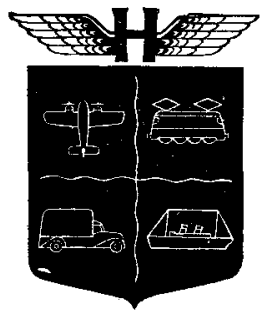

\section{Compagnie d'Assurances Générales}

\section{L'HELUETIA}

\section{ST-GALL (Suisse)}

Fondée en 1858

$\begin{array}{lc}\text { Capital social ( } 1 / 2 \text { versé) } & \text { Francs suisses } \\ \text { Total des moyens de garantie } & 61.0000 .000\end{array}$

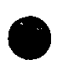

\section{ASSURAMGES TRAMSPORTS}

RÉASSURAMICES dans toutes les Branchos

Direction: ST-GALL (Suisse) Téléphone: (071) 233121

Agences en Suisse et dans beaucoup d'autres pays 


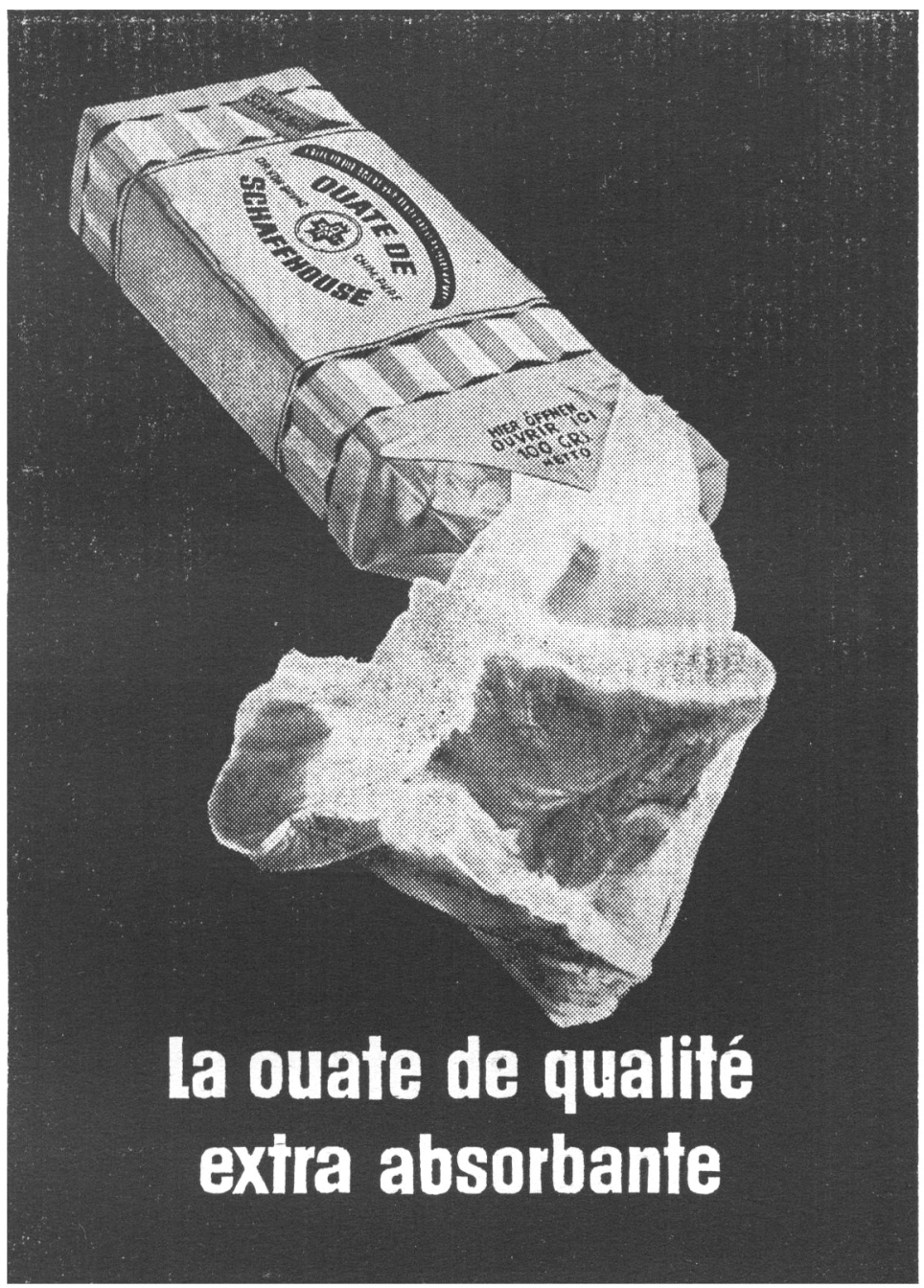



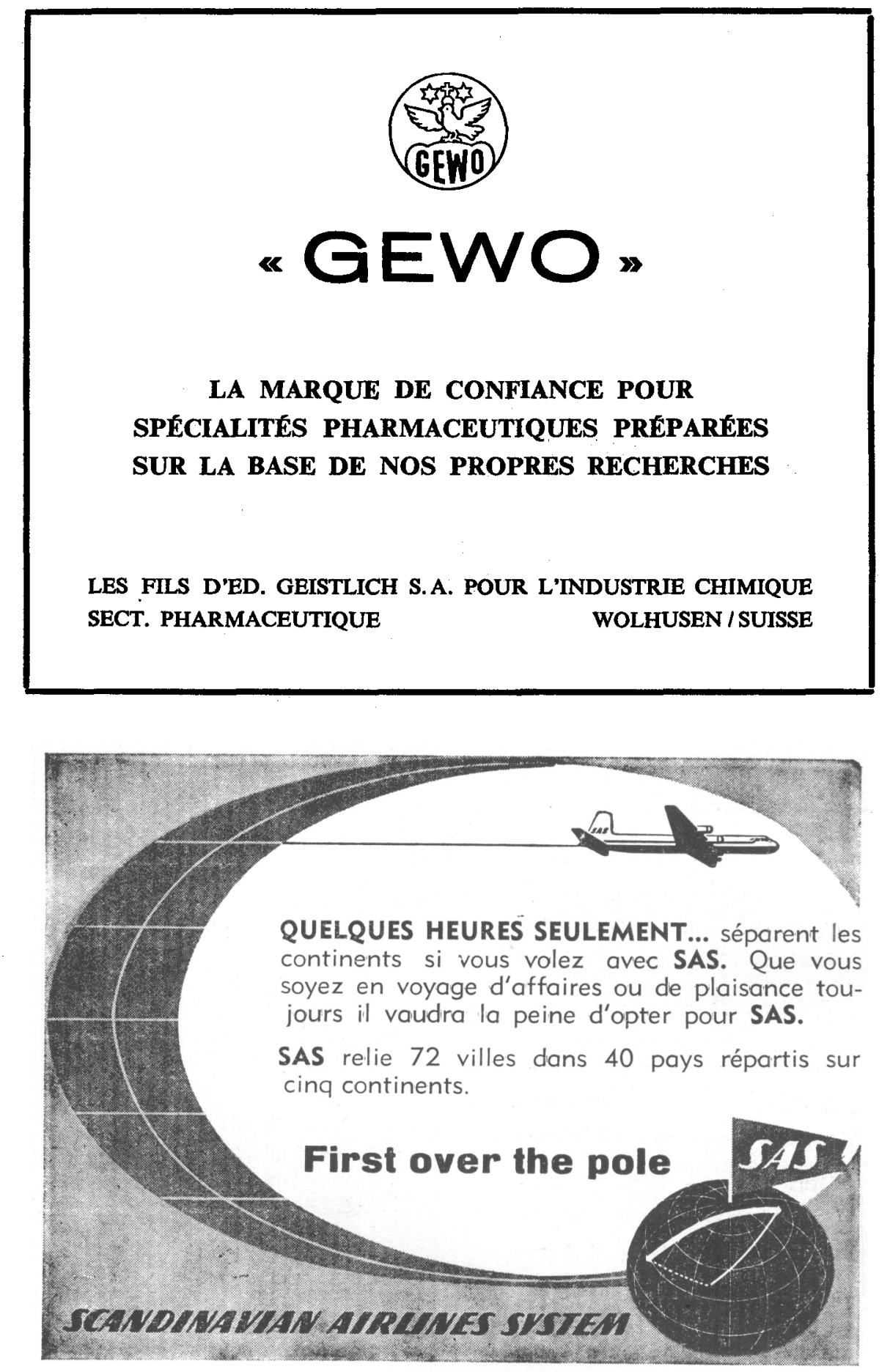

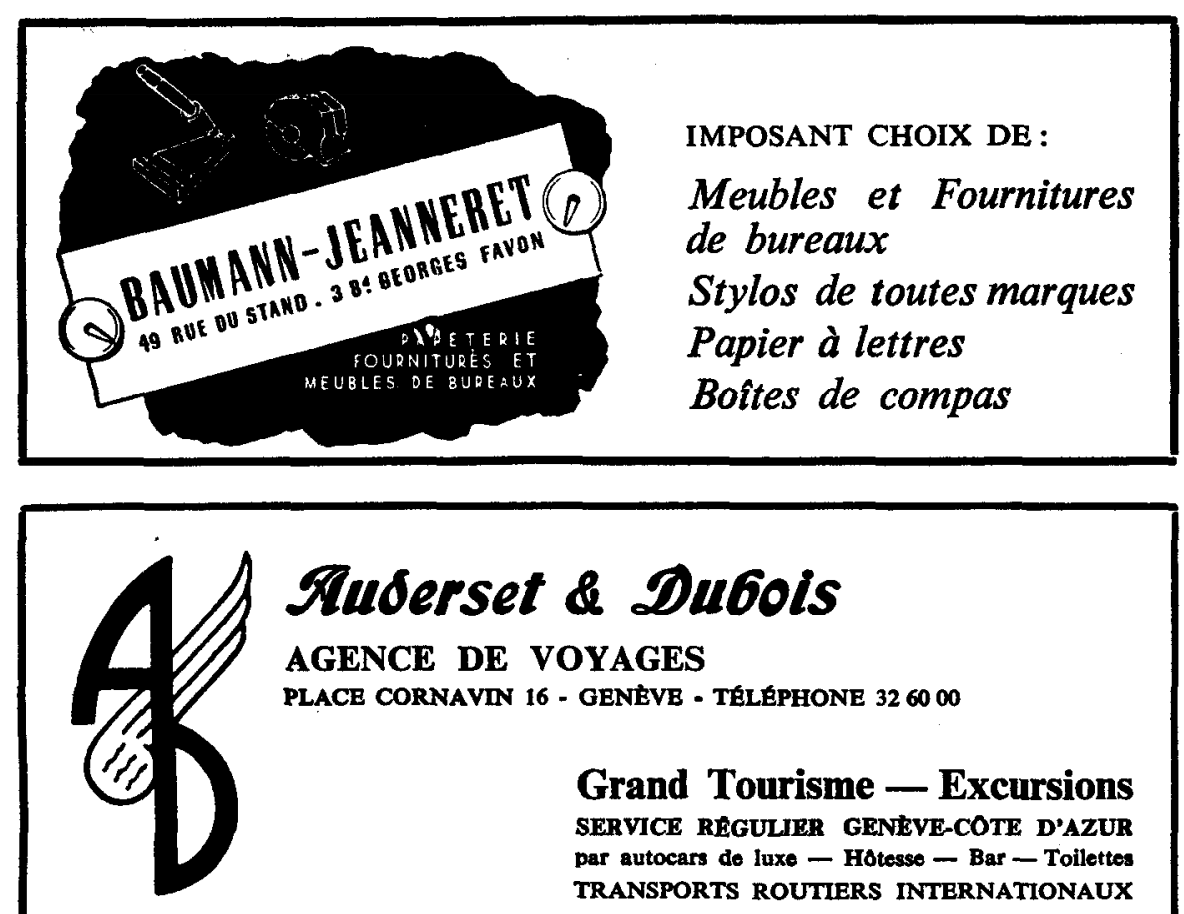

Ruderset \& Dubois AGENCE DE VOYAGES PLACE CORNAVIN 16 - GENEVE - TÉlÉPHONE 326000

Grand Tourisme - Excursions SERVICE REGULIRR GENEVE-COTE D'AZUR par autocars do luxe - Hotesse - Bar - Toilettos
TRANSPORTS ROUTIERS INTERNATIONAUX

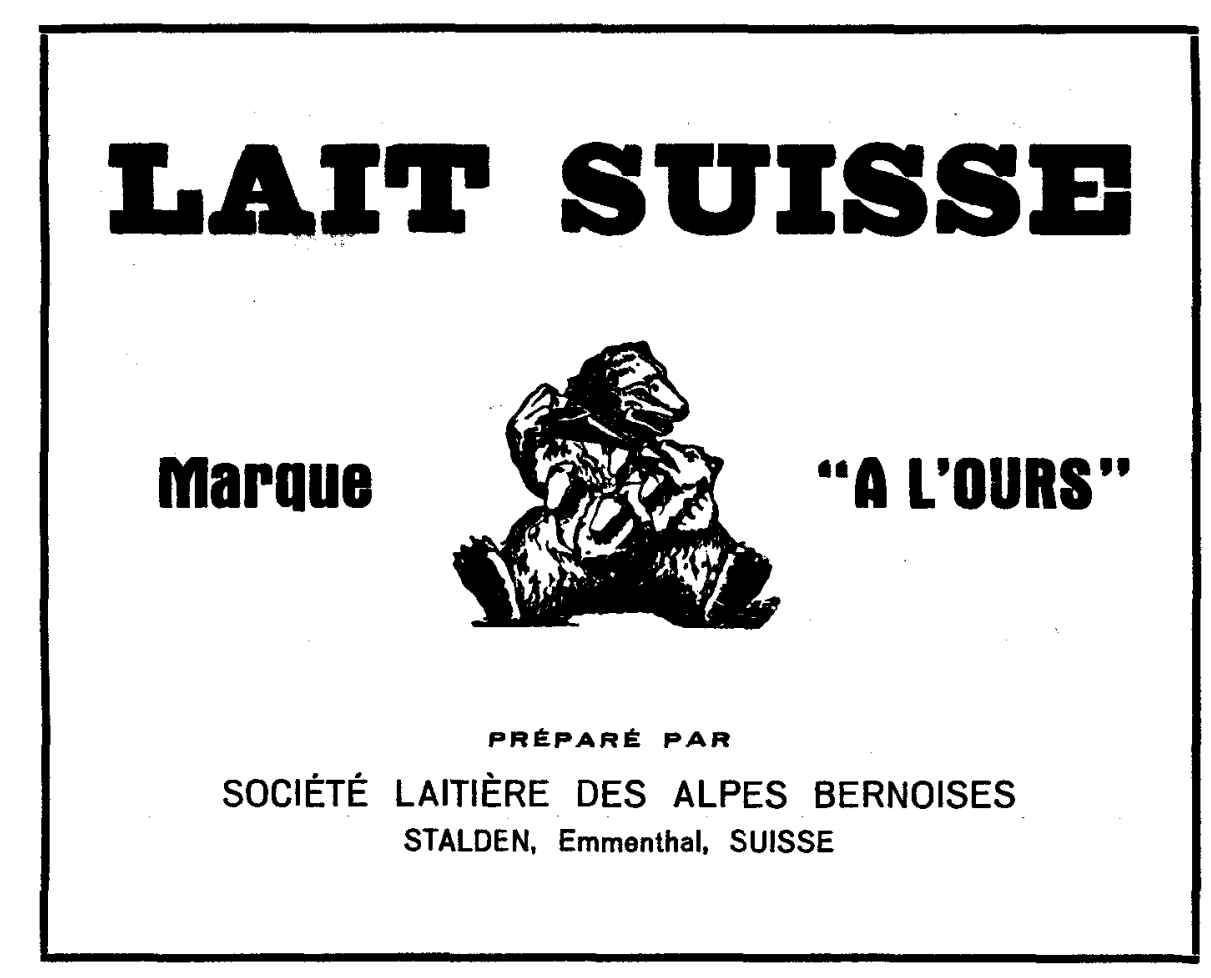

https://doi.org/10.1017/S1026881200168990 Published online by Cambridge University Press 
Nous élaborons nos spécialités pharmaceutiques avec le concours des instituts et cliniques en vue d'après les méthodes de recherches scientifiques les plus modernes

Les préparations portant la marque

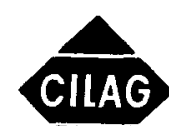

sont partout appréciées des médecins pour leur qualité éprouvée, constante et sûre

\section{CILAG SOCIÉTE ANONYME SCHAFFHOUSE}




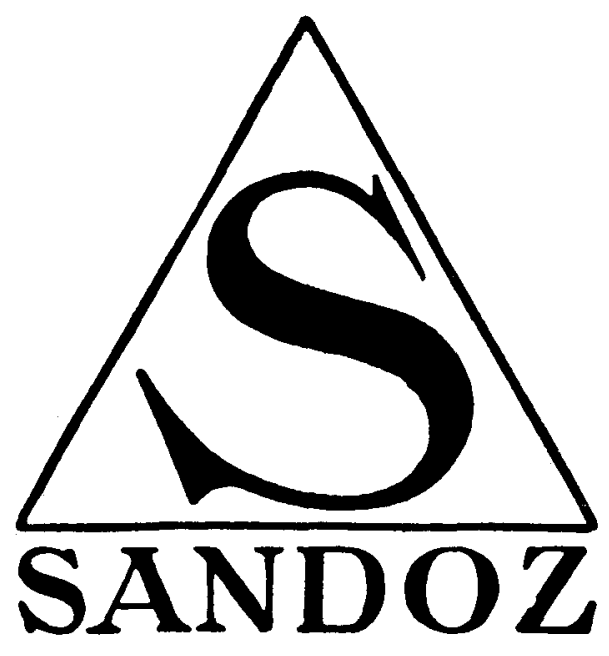

PRODUITS

\section{PHARMACEUTIQUES}

\section{BALE (Suisse)}




\section{SOCIÉTÉ ANONYME \\ FIDUGIAIRE SUISSE}

BALE

Freiestrasse 90

Zurich

Talstrasse 80

Genève

Rue du Mont-Blanc 3

Lausanne Place St-François $14 \mathrm{~B}$

ORGANISATIONS•REVISIONS • EXPERTISES

QUESTIONS FISCALES

Pour Tous Vos Voyages WAGONS-LITS//COOK

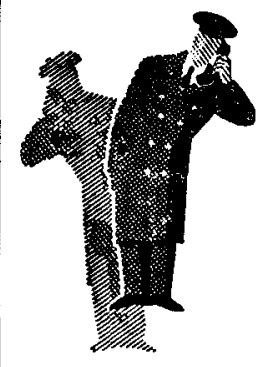

350 A GENCES à votre Service Spécialistes du Voyage à forfait Demandez nos brochures

\section{L'IMPRIMERIE} du

\section{Journal de Genève}

exécute tous travaux typographiques 


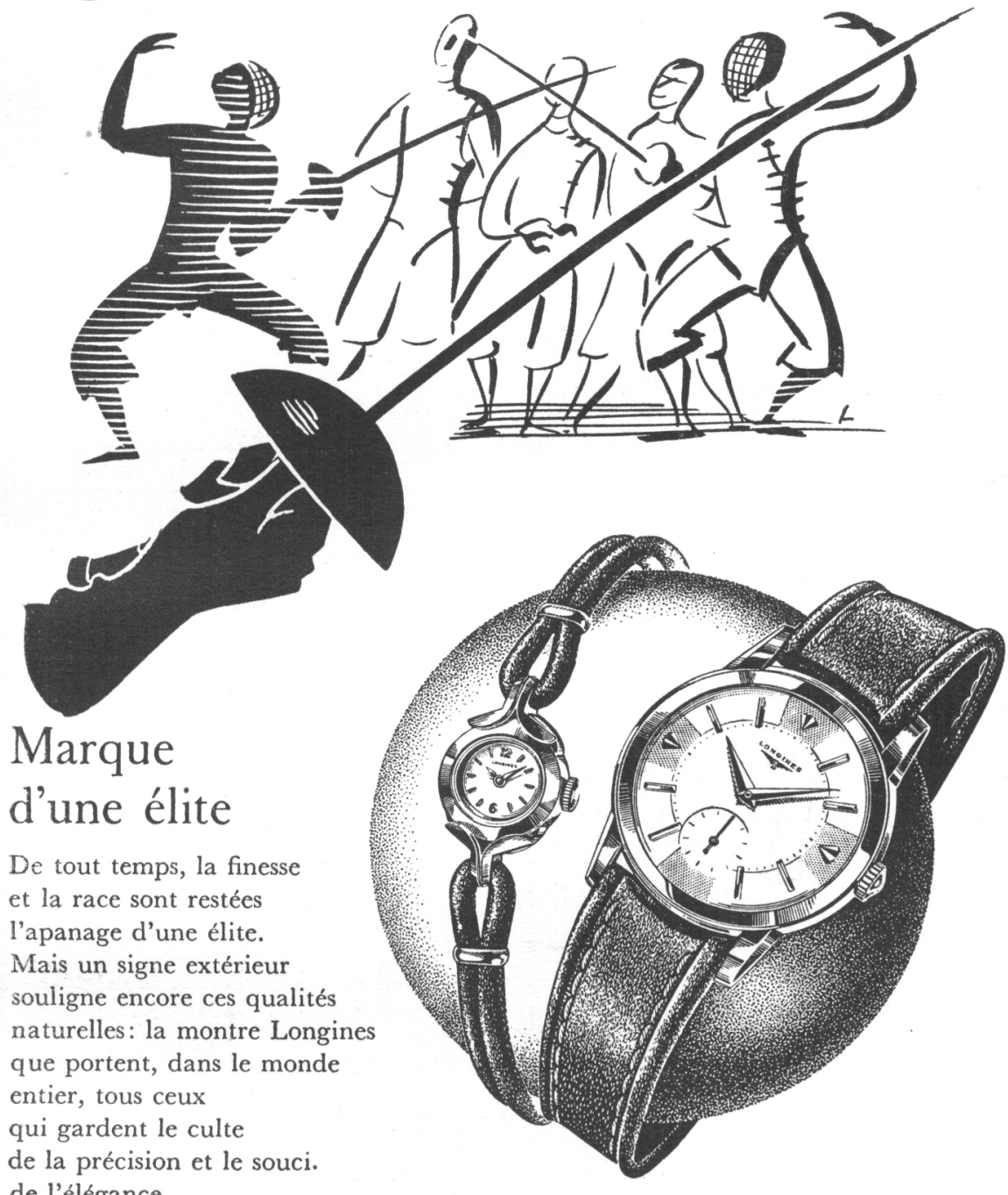

de l'élégance.

\section{LE PRIX DU TEMPS VAUT BIEN LE PRIX D'UNE}

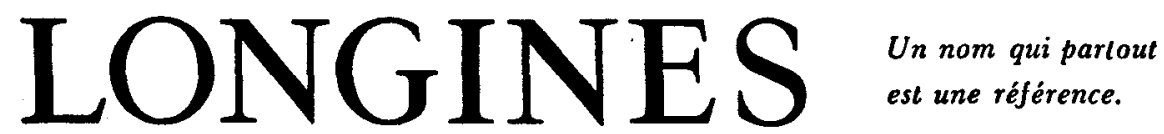




\section{SOCIÉTÉ FIDUCIAIRE ROMANDE OFOR S.A.}

- Revisions et organisations comptables

- Administration et liquidation de sociétés

- IMPOTS : Conseils par spécialiste

9, RUE D'ITALIE - GENEVE TELEPHONE 2432 91/3

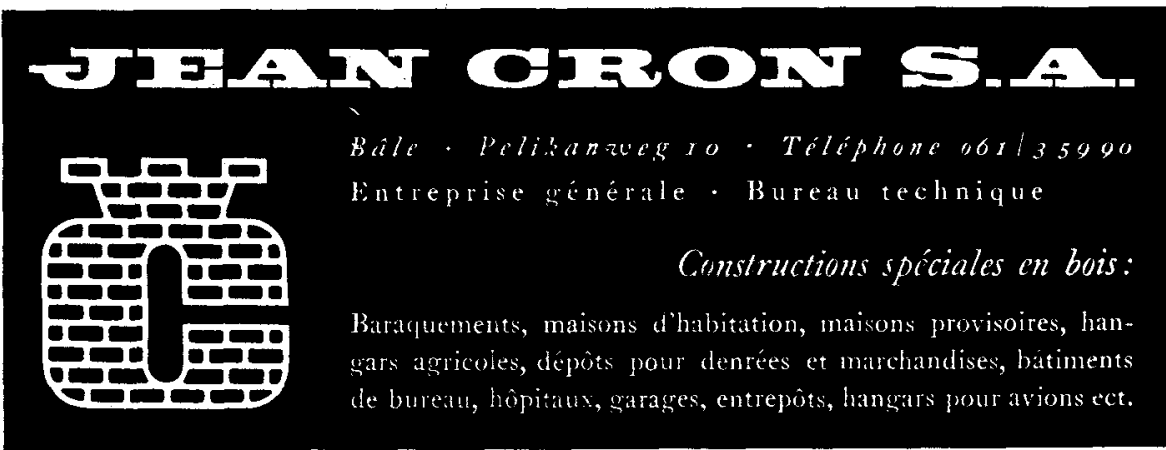

\section{GEVAERT}

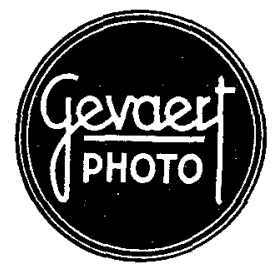

\section{CURIX - OSRAY - SCOPIX}

Le radiofilm de réputation mondiale

Photo-Produits Gevaert S. A. Belgique Représentation suisse : Gevaert Photo, Société anonyme, Bâle 6

\section{RIPASON}

extrait TOTAL de foie pour le traitement des hépatopathies chroniques

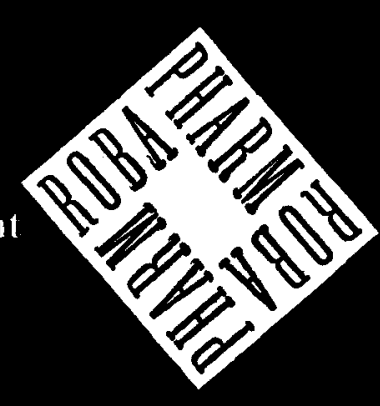




\section{ENTREPRISE GÉNÉRALE \\ ÉLECTRICITÉ ET TÉLÉPHONE}

\section{FORNASARI}

5, avenue Henri-Dunant

Tél. 244034

GEN EVE

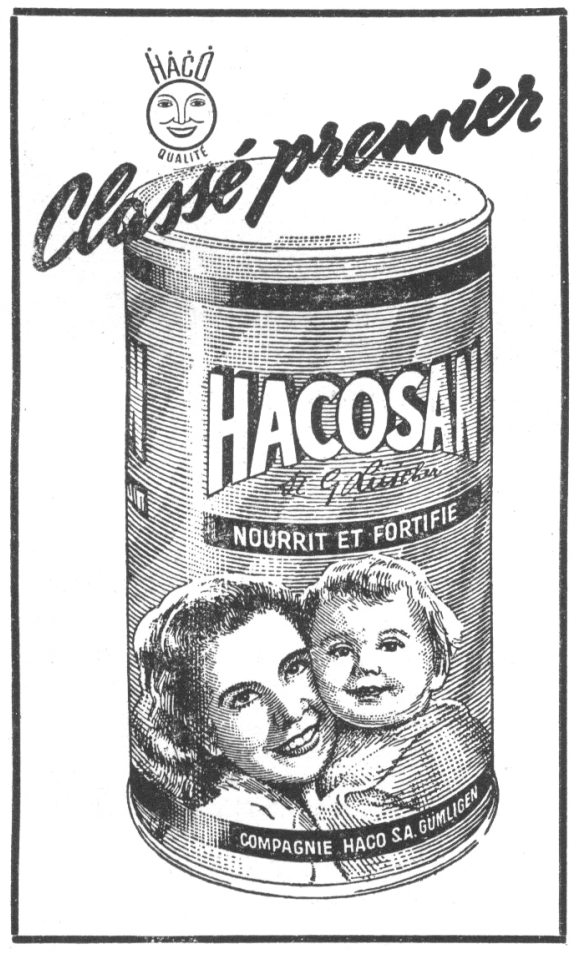

LES

LABDPATOIIFES UIFOR

S. A.

fabriquent, exportent

une gamme étendue

de produits pharmaceutiques

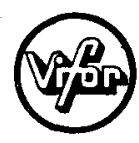

GENÈVE (Suisse) 


\section{HUGUENIN}

Mlédailleurs depuis 1868

LE LOCLE

Suls80

Insignes, Mlidailles, L्Llaquettes

en excécution artiatique

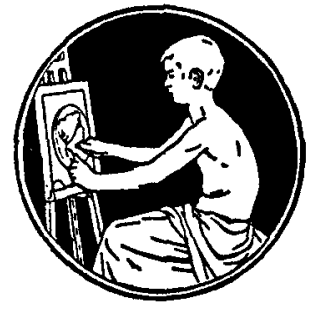

Les planches en hors-texte de la

- REVUE INTERNATIONALE DE LA CROIX-ROUGE -

sont imprimées en HCIOGRAUTE par

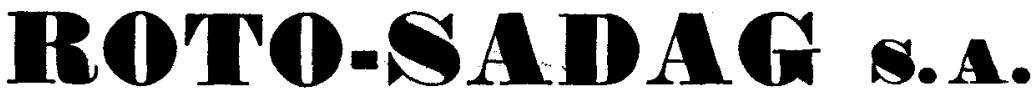

Géheron - GENEE

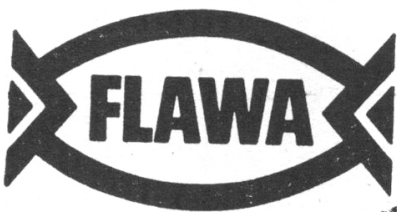

marque de qualité et de confiance pour pansements

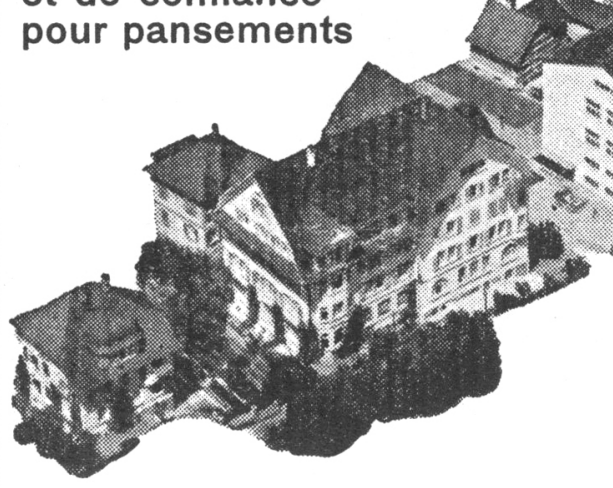

Ouates, Gazes, Bandes hydrophiles, imprégnées, stérilisées Paquets de pansement, cartouches Spécialités :

NOVA, bandes idéales élastiques DURA, bandes plâtrées

VINDEX, compresses vulnéraires VINDEX-PLAST, pansement rapide élastique MIMI, langes de gaze

CELLA, MENSA, bandes hygiéniques

FLAWA, FABRIQUES SUISSES D’OBJETS DE PANSEMENT ET D'OUATES S.A., FLAWIL, ST-GALL 